\title{
Changes of Blood Pressure in different Seasons of Bangladesh
}

\author{
MD. HARISUL HOQUE, KHURSHED AHMED, MOHAMMAD FAISAL IBN KABIR, NILUFAR FATEMA
}

Department of Cardiology, Bangabandhu Sheikh Mujib Medical University(BSMMU), Dhaka.

Address of Corespondence: Prof. Harisul Hoque, Head, Clinical Cardiology, Department of Cardiology, BSMMU, Dhaka.

E-mail: harishoque@yahoo.com

University Heart Journal 2021; 17(2): 86-90

\section{Introduction:}

Cardiovascular system is weathered, to some extent, by the seasonal changes in the environment. ${ }^{1}$ And one of the important determinants of cardiovascular diseases, related disability and death is high blood pressure. But the external factors that influence blood pressure are yet to be defined fully, although some studies have shown that BP increases significantly during winter season as compared to summer season. ${ }^{2}$ In this study, whether there is any significant difference of blood pressure in hypertensive patients in different seasons will be looked for. Bangladesh is a tropical country. Although Bangla calendar year is traditionally divided into six seasons, actually, Bangladesh has three distinct seasons: the pre-monsoon hot season from March to May, rainy monsoon season which lasts from June through October, and a cool dry winter season from November through February. There is no community based epidemiological study on the effect of season on blood pressure (BP) in adult population of Bangladesh. Different studies have shown that BP increases with decrease in temperature. ${ }^{3-11}$ Bangladesh is a tropical country. The climatic conditions in tropical areas are considerably different from those in temperate areas, with extremely hot summers and near zero temparatures during winters, resulting in an unusually large seasonal variation in temperature. In addition to geographical location, many other factors may affect the strength of the association between temperature and BP in countries like Bangladesh. A considerable proportion of the population consists of manual labourers and farmers who work in natural outdoor conditions. Housing condition and other known risk factors of hypertension (obesity, age and social stress) are different as compared to western countries, which can modify the effect of season on BP. Despite being one of the most populous areas of the world, no adequately powered study has been conducted in Bangladesh, evaluating the effect of temperature variation on BP within same subjects in general population. Thus, we had conducted a longitudinal study involving adult (18 year and above) hypertensive individuals attending the OPD of BSMMU. The aim of this study was to determine within- subject fluctuation in BP for evaluating the effect of change in season and outdoor temperature on BP.

Study place: Division of Heart Failure, Rehabilitation and Preventive Cardiology, Department of Cardiology, BSMMU, Shahbagh, Dhaka

Study population: Hypertensive Patients who came to OPD in department of Cardiology, BSMMU.

A. Study population-

a) Sample Size: Total 110 hypertensive patients

b) Age: Between 18 years and above.

Study period: $12^{\text {th }}$ October 2019 to $11^{\text {th }}$ October 2020 .

Study design: Prospective longitudinal study.

Inclusion criteria: Hypertensive Patients

Exclusion criteria:

- The patient who was not willing to participate in the study. (Non-consenting patient)

- Pregnant patients.

Method of study:

As it is a prospective longitudinal study conducted in Cardiology OPD of BSMMU. Study population was consist of hypertensive patients of both the sexes who had attended Cardiology OPD with age group of 18 years and above. Patients were randomly selected and their particulars, detailed history and physical examination and required investigations including ECG and Echo were done and recorded in a preformed data collection sheet. These patients have been followed up in the winter and in the summer. The demographic characteristics of subject were age, gender, education were recorded on a predesigned proforma. Occupation based physical activity, smoking and alcohol consumption were noted. BP was recorded using standard protocol by the doctors using sphygmomanometer. BP was recorded in surrounding which was comfortable for the patients between 9 am to 2 pm. It was assured that subjects would have sitted in a comfortable seat with no consumption of caffeine in preceding one hour or smoking in preceding $15 \mathrm{~min}$, BP devices were regularly calibrated. BP was recorded twice 
in right arm and twice in left arm in the sitting position. An average of two reading in each arm (recorded 5 min apart) was used for analysis. After the BP measurement, weight, height and waist hip ratio were measured during each season. Obesity was defined as body mass index $25 \mathrm{~kg} / \mathrm{m}^{2}$ as per the consensus statement of Asian classification ${ }^{12}$. An written informed consent was obtained from the participant.

\section{Results:}

Results A total of 125 subjects were enrolled in the study. 110 subjects were eligible for current analysis with 52 subjects in rural and 58 subjects in urban areas. Table 1 shows socio-demographic characteristics of study subjects, both rural and urban areas. Proportion of males was $55 \%$. Mean age of study population was $(43+4.50)$ years. Mean age in rural was significantly higher than urban subjects $(57+7.0$ vs $40+8.21)$ years, $p=0.04$ shows socio-demographic characteristics of study subjects, both rural and urban areas. In both genders, season had a statistically significant effect on SBP and DBP. The magnitude of the overall seasonal effect on SBP was 5.50 $\mathrm{mmHg}$ in women and $6.25 \mathrm{mmHg}$ in men. The effect of season on DBP was slightly lower in both genders: 3.50 $\mathrm{mm} \mathrm{Hg}$ in women versus $4.25 \mathrm{~mm} \mathrm{Hg}$ among men. As shown in Figure 1, the position of the overall seasonal peak (and, consequently, the position of the trough, constrained to be 6 months apart by the analytical form of the trigonometric spline) showed little variation between systolic and diastolic values and across genders. All peaks occurred in 2 weeks range in the winter, during the third and forth weeks of December, the coldest period in the year. Maximum temperatures in summer (May to July) are usually above $38^{\circ} \mathrm{c}$, and minimum temperatures in winter (November to January) below $25^{\circ} \mathrm{c}$. The differences in the position of the peak were not statistically significant. Agespecific magnitudes of seasonal effects are depicted in Figure 2. In both genders, seasonal effects on SBP increased with age, reached a maximum in the 55 to 64 years age category, and then fell among the oldest participants (65 years and above). Age-related differences in seasonal effect on DBP were much smaller, and none of them reached statistical significance. Seasonal effects were higher among urban dwellers living in informal than formal settlements, both for SBP and DBP, and for men and women.

Table-I

Demographic characteristics of study subjects, both rural and urban areas.

\begin{tabular}{lcc}
\hline Back ground of study population & $(\mathrm{N}=110)$ & Percentage \\
\hline Rural & 52 & $47.27 \%$ \\
Urban & 58 & $52.72 \%$ \\
\hline
\end{tabular}

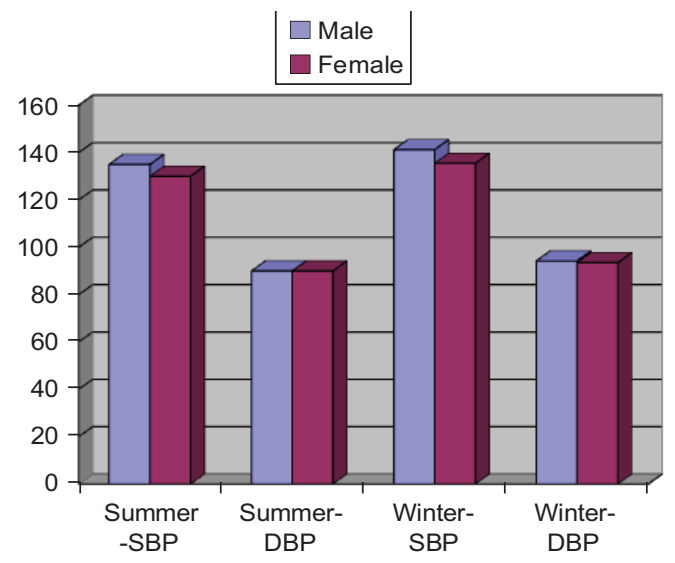

Fig.-1: Bar chart showing the difference systolic and diastolic BP during Summer and Winter.

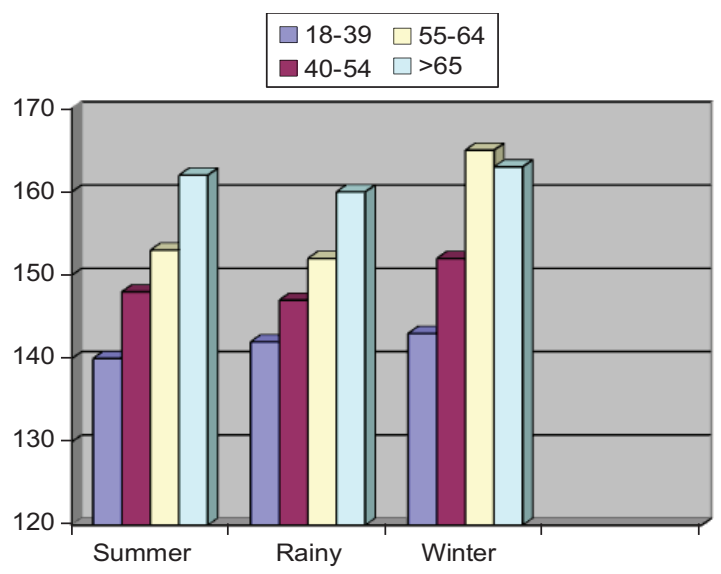

Fig.-2: In both genders, seasonal effects on SBP increased with age, reached a maximum in the 55 to 64 years age category, and then fell among the oldest participants (65 years and above).

\section{Discussions:}

The commonly observed positive relationship between magnitude of the seasonal effect and age has been confirmed in this study. This result is similar finding of a greater age effect on SBP than on DBP. ${ }^{13,14}$ In this study population, the positive trend seems to be reversed in the extreme eldest age group ( $>64$ years) which is same as result of a large cross-sectional study in China, which found that the average difference between winter and summer blood pressure increased with age up to about 70 years, and then showed a relative decrease. ${ }^{15}$ In another study, seasonal effect among older individuals (hypertensive subjects 69-91-years old), found a peak in the blood pressure among the 70 to 75 -years old, and a progressive downward trend in the subsequent age classes, suggesting that the relative reduction of the seasonal effect continues in older ages. ${ }^{16}$ The causal 
mechanism underlying this complex relationship between age and seasonal effect on blood pressure is unclear, and it is likely to reflect a combination of factors both biological and behavioral. Previous studies have shown a decrease of autonomic response to cold, ${ }^{13}$ a lower ability to control deep body temperature, ${ }^{17}$ and a reduction of the baro reflex sensitivity with increasing age. These phenomena point to a reduced ability of older subjects to compensate for the increase in blood pressure caused by cold-induced peripheral vasoconstriction. ${ }^{18}$ This physiological "mismatch" may thus produce the positive relationship between age and seasonal effect. Increased arterial wall rigidity - which has been shown to be correlated with winter-summer differences in SBP and is strongly associated with age - could also be an important causal mediator of this relationship. ${ }^{19}$ A tentative explanation of the relative decrease of winter-summer differences in the oldest age groups could be related to lower exposure to outdoor temperature by the oldest subjects because of reduced working activity and deteriorating health. In this study, the magnitude of the seasonal effect in the population as a whole was only slightly lower for diastolic than for SBP. This result is in contrast with the findings of the systematic review by Marti-Soler and other large studies, which generally indicated an average seasonal effects on DBP substantially lower than the corresponding effect on SBP. 13,20,21 This discrepancy is not unexpected, given that the populations considered in those studies were, on average, much older that the population analyzed here. As previously observed, seasonal effects on SBP increases rapidly with age, while the same trend is less evident for DBP. Therefore, in older populations the differences between diastolic and systolic effects tend to be larger than those observed in younger samples. To test the validity of this hypothesis, we recalculated seasonal effects modifying the age structure to approximately match the population studied by Su et al. ${ }^{20}$ The study of Su et al used different cut-offs to define age classes. To recreate a distribution compatible with the age categories used in our study, we hypothesized that the ages of the individual were distributed uniformly within each of the Su's classes. As expected, the ratio between seasonal effects on SBP and DBP increased from 1.1:1 to 1.6:1, closer to the 2.5:1 ratio in their study and the $2: 1$ in the review by Marti-Soler et al. Previous studies have observed that the negative relationship between outdoor temperature and blood pressure is mitigated in populations with good access to central heating at work and at home, thus suggesting socioeconomic status as a plausible effect modifier of the relationship between season and blood pressure. ${ }^{15,22}$ Our results support this hypothesis, providing evidence of an inverse relationship between magnitude of seasonal effect and education and household income, both commonly used indicators of socioeconomic status. The finding that seasonal effect is lower among urban dwellers living in formal settlements than those in informal settlements, lends some support to the hypothesis that housing conditions contribute to this effect. The effect modification appears to be stronger in women than in men, and the reasons of this discrepancy warrant further investigation. The findings of this study have implications for epidemiological, clinical, and public health practice. For epidemiological investigation, the magnitude of the seasonal effect strongly suggests that - in South Africa as in the rest of the world - studies involving the estimation of blood pressure and prevalence of hypertension should routinely take into account the period of data collection, especially when comparison with other studies is involved. Ignoring this phenomenon could bias the results, particularly if the period of data collection is restricted to a single season, as was the case of the third wave of the NIDS, where almost $50 \%$ of subjects were interviewed in winter between June and August, and none between January and March. In this case, for example, adjustment for seasonality produced a reduction of the prevalence of hypertension measured in this way among South African adults by 2 percentage points $(30.9 \%-28.9 \%)$ in males and 1.3 percentage points $(36 \%-34.7 \%)$ in females. (Adjustment for seasonality was done by randomly redistributing the period of data collection across the year, adjusting the individual values of blood pressure according to the average seasonal effect and recalculating the proportion of hypertensive subjects with the modified values of systolic and diastolic blood pressure.) In absolute numbers, the restriction of the data collection to the winter season produced an over estimation of the number of hypertensive adults by almost 600,000 , relative to the projected result were data collection spread across the seasons. (The estimation is based on the South African adult population as per Census 2011.50) count during routine clinical practice may improve the management of hypertensive (and prehypertensive) patients. The prevalence of controlled hypertension has been previously found to have a clear seasonal pattern, deteriorating in winter, which not surprisingly mirrors the fluctuations in blood pressure. ${ }^{20}$ Overall, our data support the existence of this seasonal pattern, that is, poorer control in winter than summer, suggesting that average current clinical practices are not sufficiently responsive to the seasonal modification of the patient's blood pressure levels. The 
last edition of the South African hypertension guidelines acknowledges the effects of temperature on blood pressure measurement, but makes no provision for the modification of diagnostic criteria and treatment in relation to season. ${ }^{23}$ The large seasonal variations observed especially in the older age groups suggest the need for seasonal modification of diagnostic and therapeutic clinical practice - not least because of the cited evidence of a direct correlation between winter increase of blood pressure and cardiovascular morbidity. Finally, the seasonal variations in blood pressure translated into non negligible differences by season in the projected 10-year risk of cardiovascular disease, especially in elderly subjects with low socioeconomic status where the excess risk (cumulative incidence) in winter compared with summer ranges from $2.6 \%$ (women, nonsmokers) to $5.7 \%$ (men, smokers). Although the simulations were performed mainly for illustrative purposes, and the winter increase in blood pressure may not have the same predictive meaning as long-term or chronic elevation, at population level the projection is consistent with the substantial evidence of higher cardiovascular mortality in colder months. ${ }^{24}$ In our study, the pattern of BP increases significantly during winter season as compared to summer or rainy season.

\section{Conclusions:}

This study found evidence of substantial seasonal variation in blood pressure. In both genders, seasonal variation was slightly larger for systolic than diastolic BP. Seasonal effects in SBP was significantly greater among older participants. BP increases significantly during winter season as compared to summer or rainy season. This result indicates that seasonal variations in blood pressure have concrete implications and should be routinely taken into account both in epidemiological research and in clinical practice. From a public health perspective, this findings suggest that dose adjustment of antihypertensive medication is needed to reduce older subjects' exposure to low temperatures may contribute to reduction of the winter peak in blood pressure observed. These interventions may prevent the increase cardiovascular risk in this population. Besides our findings we also suggest further study to search for possible bio-proactive component present in environment which may change their nature and configuration causing vascular endothelial to constrict or dilate.

\section{References:}

1. Sheth T, Nair C, Muller J, Yusuf S. Increased winter mortality from acute myocardial infarction and stroke: the effect of age. J Am Coll Cardiol 1999;33(7) : 1916-19.
2. Goyala A, Aslama N, Kaurb S, Sonic RK, Midhac V, Chaudhary A, Dhaliwald LK, Singha B, Chabraa ST, Mohana B, Anande IS, Wandera GS, Factors affecting seasonal changes in blood pressure in North India: A population based four seasons study. Indian Heart Journal 2018; 70 (3): 360-67.

3. Aubiniere- Robb L, Jeemon P, Hastie CE, et al. Blood pressure response to patterns of weather fluctuations and effect on mortality. Hypertension 2013; 62 (1): 190-96.

4. Su D, Du H, Zhang X, et al. Season and outdoor temperature in relation to detection and control of hypertension in a large rural Chinese population. Int J Epidemiol 2014; 43 (6): 1835-45

5. Lewington S, Li L, Sherliker P, et al. Seasonal variation in blood pressure and its relationship with outdoor temperature in 10 diverse regions of China: the China Kadoorie Biobank. J Hypertens 2012; 30 (7): 1383-91.

6. Madsen C, Nafstad P. Associations between environmental exposure and blood pressure among participations in the Oslo Health Study (HUBRO). Eur J Epidemiol 2006; 21: 485-91

7. Barnett AG, Sans S, Salomaa V, Kuulasmaa K, Dobson AJ. The effect of temperature on systolic blood pressure.

8. Jenner DA, English DR, Vandongen R, Beilin LJ, Armstrong BK, Dunbar D. Environmental temperature and blood pressure in 9-year-old Australian children. J Hypertens 1987; 5: 683-86.

9. Sega R, Cesana G, Bombelli M, et al. Seasonal variations in home and ambulatory blood pressure in the PAMELA population. Pressure Arteriose Monitorate Eloro Association J Hypertens 1998; 16: 1585-92.

10. Modesti PA. Season, temperature and blood pressure: a complex interaction. Eur J Intern Med 2013; 10. 1016/j.ejim 2013.08.002.

11. Modesti PA, Rapi S, Bamoshmoosh M, et al. Impact of one or two visits strategy on hypertension burden estimation in HYDY, a population-based cross- sectional study: implications for heathcare resource allocation decision making BMJ Open 2012; 2 (4): e 00106210. 1136/ bmjopen-2012-001062.

12. Misra A, Chowbey P, Makkar BM, et al. For consensus group: Consensus statement for diagnosis of obesity and the metabolic syndrome for Asian Indians and recommendations for physical activity, medical and surgical management. J Assoc Phys India 2009; 57:163-70.

13. Tu Y-K, Chien K-L, Chiu Y-W, et al. Seasonal variation in blood pressure is modulated by gender and age but not by BMI in a large Taiwanese population, 1996-2006. J Am Soc Hypertens. 2013;7:216-28.

14. Alpe'rovitch A, Lacombe J-M, Hanon O, et al. Relationship between blood pressure and outdoor temperature in a large sample of elderly individuals: the Three-City study. Arch Intern Med. 2009;169:75-80.

15. Lewington $\mathrm{S}, \mathrm{Li} \mathrm{L}$, Sherliker P, et al. Seasonal variation in blood pressure and its relationship with outdoor temperature in 10 diverse regions of China: the China Kadoorie Biobank. J Hypertens. 2012;30:1383-91. 
16. Charach G, Rabinovich PD, Weintraub M. Seasonal changes in blood pressure and frequency of related complications in elderly Israeli patients with essential hypertension. Gerontology. 2004;50:315-21.

17. Collins KJ, Exton-Smith AN, James MH, et al. Functional changes in autonomic nervous responses with ageing. Age Ageing. 1980;9:17-24.

18. Kingma BRM, Frijns AJH, Saris WHM, et al. Cold-induced vasoconstriction at forearm and hand skin sites: the effect of age. Eur J Appl Physiol. 2010;109:915-21.

19. Youn J-C, Rim S-J, Park S, et al. Arterial stiffness is related to augmented seasonal variation of blood pressure in hypertensive patients. Blood Press. 2007;16:375-80.

20. Su D, Du H, Zhang X, et al. Season and outdoor temperature in relation to detection and control of hypertension in a large rural Chinese population. Int J Epidemiol. 2014; 43:1835-45.

21. Marti-Soler H, Gubelmann C, Aeschbacher S, et al. Seasonality of cardiovascular risk factors: an analysis including over 230 000 participants in 15 countries. Heart. 2014;100:1517-23.

22. Kristal-Boneh E, Harari G, Green MS, et al. Seasonal changes in ambulatory blood pressure in employees under different indoor temperatures. Occup Environ Med. 1995;52:715-21.

23. Seedat YK, Rayner BL. South African hypertension guideline 2011. S Afr Med J. 2012;102 (1 Pt 2):57-83.

24. Marti-Soler H, Gonseth S, Gubelmann C, et al. Seasonal variation of overall and cardiovascular mortality: a study in 19 countries from different geographic locations. PLoS One. 2014;9:e113500. 